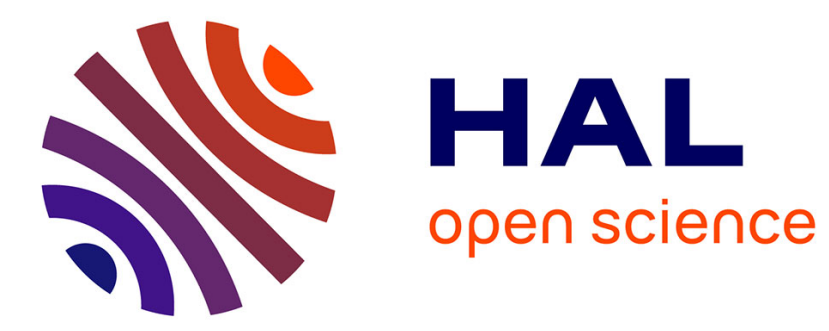

\title{
Nonlinear Darcy flow dynamics during ganglia stranding and mobilization in heterogeneous porous domains
}

\author{
A. Yiotis, A. Dollari, M.E. Kainourgiakis, Dominique Salin, Laurent Talon
}

\section{To cite this version:}

A. Yiotis, A. Dollari, M.E. Kainourgiakis, Dominique Salin, Laurent Talon. Nonlinear Darcy flow dynamics during ganglia stranding and mobilization in heterogeneous porous domains. Physical Review Fluids, 2019, 4, 10.1103/PhysRevFluids.4.114302 . hal-03022270

\section{HAL Id: hal-03022270 \\ https://hal.science/hal-03022270}

Submitted on 25 Nov 2020

HAL is a multi-disciplinary open access archive for the deposit and dissemination of scientific research documents, whether they are published or not. The documents may come from teaching and research institutions in France or abroad, or from public or private research centers.
L'archive ouverte pluridisciplinaire HAL, est destinée au dépôt et à la diffusion de documents scientifiques de niveau recherche, publiés ou non, émanant des établissements d'enseignement et de recherche français ou étrangers, des laboratoires publics ou privés. 


\title{
Nonlinear Darcy flow dynamics during ganglia stranding and mobilization in heterogeneous porous domains
}

\author{
A. G. Yiotis, ${ }^{*}$ A. Dollari, ${ }^{\dagger}$ and M. E. Kainourgiakis \\ Environmental Research Laboratory, National Center for Scientific Research "Demokritos," \\ 15341 Agia Paraskevi, Greece \\ D. Salin and L. Talon \\ Laboratoire FAST, Université Paris Saclay, 91405 Orsay Cedex, France
}

(Received 19 June 2019; published 19 November 2019)

\begin{abstract}
We study the steady-state displacement of nonwetting liquid ganglia during immiscible two-phase flows in realistic, stochastically reconstructed porous domains, focusing primarily on the nonlinear Darcian regime that arises when capillary to viscous (or gravity) forces become comparable at the pore scale. During this process, the ganglia undergo a continuous cycle of dynamic coalescence and fragmentation, resulting in two populations (a mobile and a stranded one) with distinct structural and rheological features, that continuously exchange mass between them under "stationary" flow conditions. We use a lattice Boltzmann model for the explicit solution of flow and interfacial dynamics at the pore scale driven by a constant body force field (i.e., gravity), and a periodic clustering algorithm for the identification and classification of mobile and stranded ganglia. Our simulation results reveal that an increase in the applied Bond number (Bo) leads to a gradual mobilization of the initially stranded ganglia population, resulting in a power-law scaling with an exponent being a strong function of the nonwetting-phase saturation. The linear Darcian scaling for the nonwetting phase is progressively restored at high Bo, while the wetting phase appears to maintain a linear Darcian scaling over the entire range of Bo values. We show that the mobilization process is characterized by a critical Bo, which is independent of saturation, above which new flow paths are created, in a similar fashion as a yield-stress fluid flows in a porous medium. Our results also offer a unique insight on the distinct structural characteristics of the mobile and stranded populations (e.g., ganglia size and length), as well as on their velocity and orientation with respect to their size.
\end{abstract}

DOI: 10.1103/PhysRevFluids.4.114302

\section{INTRODUCTION}

The flow of non-aqueous-phase liquids (NAPLs) in the form of disconnected droplets or ganglia within macroporous media is a ubiquitous process in geologic, environmental, and energy-related applications. These include, among others, the recovery of stranded oil residuals in petroleum reservoirs during enhanced oil recovery operations [1], the remediation of soils from insoluble anthropogenic pollutants [2], and the permanent trapping and storage of supercritical $\mathrm{CO}_{2}$ in deep saline aquifers [3]. In recovery applications, the mobilization of such nonwetting-phase (nw-phase) residuals, stranded due to capillary forces and flow by-pass in lower permeability regions of the

*Corresponding author: School of Mineral Resources Engineering, Technical University of Crete, 73100 Chania, Greece; yiotis@mred.tuc.gr

'Present address: Laboratoire FAST, Université Paris Saclay, 91405 Orsay Cedex, France. 


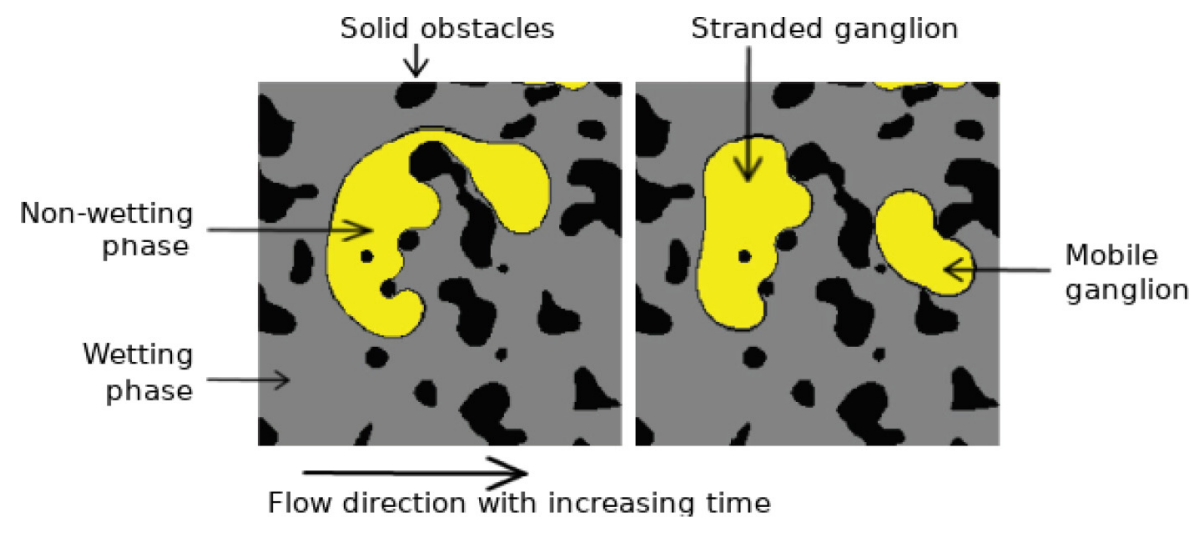

FIG. 1. A schematic of a ganglion breakup (fragmentation) event during immiscible two-phase flow within a disordered two-dimensional (2D) porous domain, as it is visualized at the pore scale. This event generates a stranded ganglion in the upstream region and a mobile one that flows further downstream driven by the surrounding w-phase. The ganglia (nw-phase) are shown in yellow (brighter color in gray scale), the w-phase in gray, and the solid sites in black.

porous structure, is typically achieved by the injection of a purge wetting fluid (w-phase), in a process that can be visualized as secondary imbibition. Due to the disconnected nature of such residuals, the dominant physics controlling ganglia movement arises at the pore scale, where local viscous forces that drive flow compete with capillary forces that impose ganglia stranding within pore-space confinements. When local flow conditions are favorable, the normal and shear stresses exerted by the flowing w-phase over ganglia interfaces results in their mobilization and advection along the flow path $[4,5]$.

Ganglia mobilization at increasing flow rate conditions is typically accompanied by their dynamic breaking up (i.e., fragmentation) into smaller ones. This pore-scale process occurs when viscous drag from the continuous w-phase significantly overcomes local interfacial tension [6-8], causing the interface of mobile ganglia to deform as they "squeeze" through narrower pore configurations (a process also known as snap-off; see, e.g., Fig. 1) [9]. The generation of smaller ganglia (with typical sizes on the order of the average pore size) will in turn lead to their temporary stranding further downstream within the porous structure, either until local flow conditions become again favorable for their remobilization or until they coalesce with larger mobile ones [10,11].

This complex dynamic process of ganglia fragmentation and coalescence, accompanied by continuous cycles of mobilization and stranding within the tortuous and heterogeneous void space of macroporous media, is not well understood primarily due to technological limitations in the visualization of ganglia dynamics within actual three-dimensional (3D) porous materials under realistic flow conditions. A series of elaborate experimental studies have thus focused on simplified 2D permeable structures, such as individual capillaries [12], 2D pore networks [5,9,13,14], and monolayers of glass beads $[11,15]$. These studies have provided significant insight on the complex local flow dynamics that lead to ganglia mobilization and breaking up, revealing also the important effects of capillarity [16], viscosity ratio [13], and flow history [14], among many other parameters, on the residual ganglia saturation. Much more limited is the experimental work performed in more realistic 3D domains, such as sand packings [17,18], glass bead packings [16,19,20], sintered glass filters [21,22], and more recently in actual porous rocks [23-26], which were realized using state-of-the-art visualization technologies, such as X-ray micro-CT and confocal microscopy. The latter studies have focused primarily on the resulting size distributions of residual (i.e., stranded) ganglia saturations after flushing the porous sample over several imbibition cycles. The dynamics of ganglia coalescence and breaking up during such processes are typically not recovered due to the limited temporal resolution of such methods. 
In parallel, experimental work in this field has been supported by theoretical and numerical models relying either on mean-field approximations of the process dynamics [11,27] or rigorous pore-network models [13,28-31], as well as combinations of both. In a seminal contribution, $\mathrm{Ng}$ and Payatakes [32] provided the basis for the stochastic approximation of ganglia flow in porous media based on locally defined mobilization and stranding probabilities, while also considering the breaking up and coalescence rates as random events which were, however, expressed as a function of ganglia size. This approach has been extended by more elaborate stochastic models, such as those of Darcian dynamics by Amili and Yortsos [33], mechanistic models relying on the consideration of "prototype" flows [34,35], and a probability density function approach in the framework of continuous Markov processes by Tyagi and Jenny [36]. While providing invaluable information for the coupling between microscale physics and field scale behavior, a common characteristic of the above approaches is that they rely on microscale probabilities in the form of mechanistic rules that need to be resolved by either rigorous pore-scale experiments or the direct solution of flow and/or conservation equations for immiscible flow at this scale.

Such studies have highlighted some important scaling relations for the ganglia size with respect to the capillary number, $\mathrm{Ca}$, i.e., the ratio of viscous to capillary forces that control the movement of interfaces at the pore scale. The ganglia size distribution is typically found to scale as $p(s) \propto$ $s^{-\tau} \exp \left(-s / s^{*}\right)$, where $\tau$ is a scaling exponent, $s$ is the ganglion size, and $s^{*}$ is the maximum ganglion size that is found to scale as $s^{*} \propto \mathrm{Ca}^{-\zeta}$. The exponents $\tau$ and $\zeta$ have been experimentally determined to be $\tau \approx 2.07$ and $\zeta \approx 1[14,15,25]$, without, however, an explicit distinction between mobile and stranded ganglia populations. Furthermore, previous studies have demonstrated the existence of a nonlinear Darcian regime, where the total flow rate follows a power-law scaling with the applied pressure drop $q \propto(\Delta P)^{\beta}$, with $\beta$ being an exponent that depends on the experimental protocol and which is found to be in the range 1.4-2.0 [15,27].

While the physical mechanism responsible for such a divergence from the classic Darcian linear scaling is still not yet fully understood, a common consensus is that it is due to the gradual mobilization of otherwise stranded ganglia at increasing Ca flow conditions. Ganglia mobilization alters the effective permeability of the medium by allowing fluid flow from previously blocked flow paths [10,11,27]. In this context, Sinha and Hansen [27] highlighted the similarity of this process to the flow of yield-stress (Bingham) fluids in porous media [37,38], where new flow paths are created as a critical stress value (which is a function of the local pore size) is exceeded. For such a process, it has been shown that the total flow rate follows a quadratic relationship with the applied pressure difference, where the power-law exponent appears to be quite robust and independent of the disorder characteristics of the medium. In a similar fashion the physical origin of the nonlinear scaling for ganglia dynamics appears to emanate at the pore scale due to the formation of new preferential flow paths resulting from ganglia mobilization when a critical local pressure difference is achieved. Using a mean-field approach Sinha and Hansen [27] proposed a power-law exponent of 2.0 for the nonlinear regime. A possible flaw, however, of their argument, that could also explain the divergence with the experimentally reported scaling exponents, is the consideration that the porous medium is homogeneously filled with an "effective" yield-stress fluid.

In this contribution, we study numerically the effective rheology of the specific two-phase flow process (particularly within the nonlinear Darcian regime) in realistic, stochastically reconstructed 2D porous domains, focusing primarily on the discrete contributions of the mobile and stranded ganglia populations and their characteristics. Our main objective is to highlight the physical origin of the reported power-law dependence and quantify the contribution of each phase in the resulting flow dynamics. For this purpose, we use a lattice Boltzmann (LB) scheme that solves explicitly for immiscible two-phase flow and interfacial dynamics at a very fine (sub-pore-scale) resolution within the disordered void space of the medium. This approach can be applied to extremely structurally complex domains without the need to explicitly define "mechanistic" or "stochastic" rules for the pore-scale behavior of fluid interfaces at the expense, however, of significant computational resources and simulation times [10]. 
Assuming a periodic 2D domain in all directions and an appropriately selected volume-averaged intrinsic velocity threshold to distinguish stranded from mobile ganglia, we simulate the coexistence of both mobile and stranded ganglia populations, and resolve the dynamic repartitioning of mass between them through breaking up and coalescence at steady-state flow conditions. The ratio of mobile to stranded saturations is thus a dependent variable at a fixed nw-phase saturation value, unlike typical earlier experimental studies, where the nw-phase mass is recovered from the medium at increasing $\mathrm{Ca}$, starting with the mobilization and removal of larger ganglia [14,20], and thus only stranded ganglia are accounted for. Such a configuration appears in very few experimental studies where steady-state flow is achieved through the co-injection of both fluids at fixed ratios $[5,11,16,39]$. These works, however, fail to distinguish between mobile and stranded ganglia populations and report their individual characteristics. Thus the previously reported values for the scaling exponent of the nonlinear Darcy regime typically refer to the combined Darcy flow of both phases.

\section{NUMERICAL MODEL}

In the present study, we use a LB simulator for the solution of two-phase flow through a stochastically reconstructed and disordered porous domain. Our numerical model relies on the two equation diffuse-interface model originally proposed by $\mathrm{He}$ et al. [40], appropriately modified to account also for capillarity using an alternative forcing scheme (as described in our previous works $[10,41])$, and a series of robust numerical stabilization strategies, proposed initially by Lee and co-workers $[42,43]$. This model effectively recovers the incompressible Navier-Stokes equation with an additional forcing term that accounts for interfacial tension and wettability, coupled with an evolution equation that tracks the convective interface motion, in a similar fashion as the level set and phase field diffuse interface methods (see, e.g., Ref. [44]), or other LB schemes [45,46]. For a detailed description of the LB formulation used in this study, the reader is referred to Refs [10,41].

The porous medium is represented by $2 \mathrm{D}$ domains in the form of discrete and periodic permeability fields of size $L^{2} \delta x^{2}$, where $\delta x$ is the lattice space unit. Each domain consists of solid (impermeable to flow) and void (permeable) sites, where the spatial distribution of the solid sites is accomplished using a standard spectral method that pursues the recovery of the statistical properties of naturally occurring porous media, such as sandstone. In our case, these properties are the porosity, $\phi$, and the two-point autocorrelation function, parametrized by the correlation length $\lambda_{s}$, for the spatial distribution of the solid phase $[47,48]$. Such a discrete periodic 2D domain is shown in Fig. 2(a). A detailed description of the stochastic reconstruction algorithm used in this study can be also found in Ref. [10].

It is straightforward to show that the superficial (Darcy) velocity of a Newtonian liquid flowing in such a domain is proportional to the applied body force $\vec{F}$ by solving the previously described LB scheme under single-phase flow conditions (namely, $S_{w}=1$ ). The intrinsic permeability of the medium $k$, determined by Darcy's law, is also found to scale with the correlation length, $k \propto \lambda_{s}^{2}$, and thus the parameter $\lambda_{s}$ can be used as a proxy for the average pore size.

\section{NUMERICAL SIMULATIONS}

We performed a series of numerical simulations in 2D reconstructed porous domains to study the effects of the flow rates and the nw-phase (ganglia) saturation, $S_{n w}$, on the rheological behavior of the system and the distinct characteristics of the resulting mobile and stranded ganglia populations. In all our simulations, the nw-phase is initially randomly distributed in the void space using the same stochastic algorithm as the one for the solid phase, and a correlation length of $\lambda_{n w}=30 \delta x$. All simulations are performed over three different random domains of size $2048 \times 2048 \delta x^{2}$, however, with the same correlation length, $\lambda_{s}=15 \delta x$, and the same porosity, $\phi=0.7$. Such a high value for the porosity is required to ensure the existence of a sufficient number of percolating flow paths across the $2 \mathrm{D}$ periodic domain. 


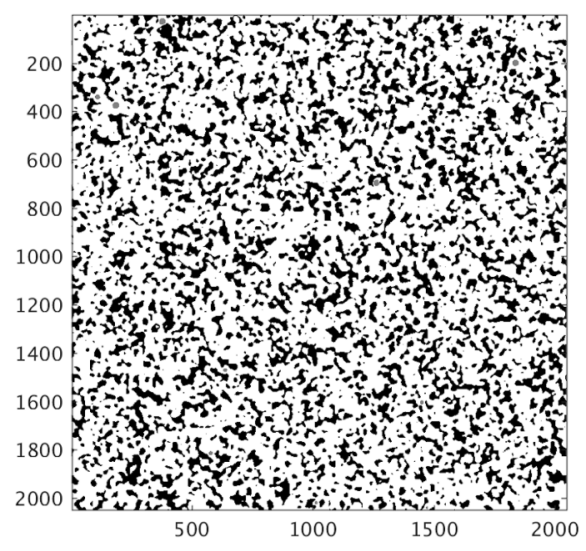

(a)

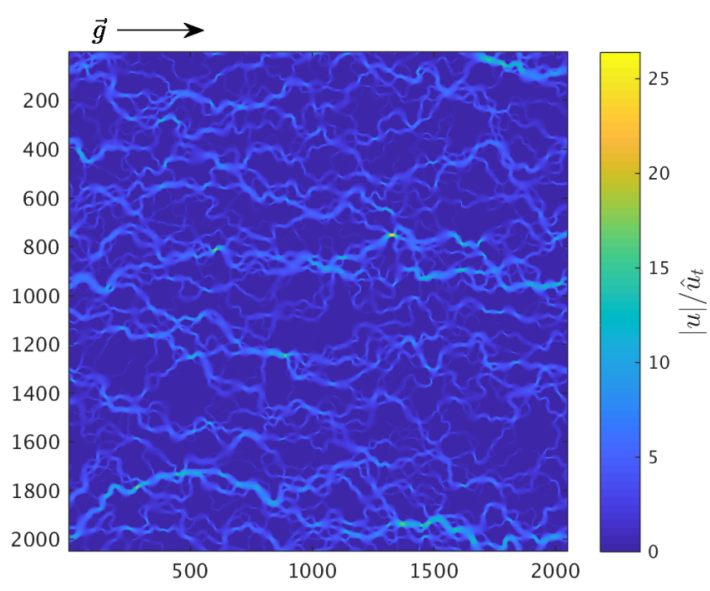

(b)

FIG. 2. (a) A typical reconstructed 2D porous domain used in this study. The black and white colors denote solid (impermeable to flow) and void (permeable) space, respectively. (b) Scaled velocity magnitude, $|u| / \hat{u}_{t}$, during creeping single-phase flow within the domain. Lighter colors correspond to higher velocities, while darker blue colors correspond to solid sites or flow stagnation regions. The calculated intrinsic permeability of the domain is $k=9.9 \delta x^{2}$. Reported lengths are in lattice space units $[\delta x]$.

A constant body force, $\vec{F}=\rho \vec{g}$, where $\rho$ is the fluid density and $\vec{g}$ is the acceleration of gravity, is then applied to generate flow, while periodic flow conditions are considered along all four sides. This particular approximation was selected over a fixed pressure difference due to the technical complexity of applying pressure boundary conditions across the sides of periodic domains, where two-phase flows occurs. Our approach is, however, exact for gravity-driven flows at the pore scale, while we expect that it also holds at the much coarser representative elementary volume (REV) scale.

By initially performing a single-phase flow simulation, the intrinsic permeability of the medium was found to be practically equal to $k=9.9 \delta x^{2}$ for all three domains. Figure 2(a) shows one of the reconstructed domains used in this study, while Fig. 2(b) shows the calculated velocity magnitude for creeping single-phase flow with a body force applied in the $x$ direction. The dominant flow paths that span the entire periodic domain in the $x$ direction appear in this figure with lighter blue color, while the solid phase [corresponding to the black sites of Fig. 2(a)] and the stagnant flow regions appear in darker blue color. For two-phase flow within the same domain, we expect that the presence of the stranded ganglia will block some of these flow paths, thus effectively decreasing the relative permeability of the domain.

The flow process is then characterized by two dimensionless numbers: the capillary number, $\mathrm{Ca}=\hat{u}_{t} \mu_{e} / \gamma$, which expresses the ratio of viscous to capillary forces across interfaces, and the Bond number, Bo $=|\vec{g}|\left(\rho_{w}-\rho_{n w}\right) \lambda_{s}^{2} / \gamma$, which expresses the ratio of gravity to capillary forces at the pore scale. Here, $\rho_{w}$ and $\rho_{n w}$ are the densities of the w- and nw-phases, respectively, $\gamma$ is the fluid-fluid interfacial tension, $\mu_{e}$ is an effective saturation-weighted viscosity, defined as $\mu_{e}=$ $S_{n w} \mu_{n w}+\left(1-S_{n w}\right) \mu_{w}$, and $\hat{u}_{t}=\hat{u}_{w}+\hat{u}_{n w}$ is the total superficial (Darcy) velocity of both fluids, where

$$
\hat{u}_{i}=\frac{1}{A} \int_{A}\left(\vec{u}_{i} \cdot \vec{n}\right) d A, \quad i=w, n w .
$$


The equivalent interstitial (pore-scale) velocity for each phase is defined as

$$
u_{i}=\frac{1}{A_{i}} \int_{A_{i}}\left(\vec{u}_{i} \cdot \vec{n}\right) d A, \quad i=w, n w .
$$

In the above equations, $\vec{n}=\frac{\vec{g}}{|\vec{g}|}$ is the unit vector in the direction of the applied body force and $\vec{u}_{i}$ is the local fluid velocity vector for each phase, $i=w, n w$. Note that $A=A_{w} \cup A_{n w} \cup A_{s}$, where $A_{w}$, $A_{n w}$, and $A_{s}$ are the domain sites occupied by the wetting, nonwetting, and solid phases, respectively.

In all our simulations, we keep the fluid densities and the interfacial tension as constants, while $\vec{g}$ is the real control parameter. Thus the resulting Bo is used as a proxy for the applied pressure difference at the REV scale, and Ca is a dependent variable. Here, we take a value for $\rho_{n w}=0.579 \rho_{0}$ and $\rho_{w}=1.461 \rho_{0}$, where $\rho_{0}$ is a reference density in our system. This is a convenient choice to match the density ratio of an oil/aqueous system, while also taking advantage of the underlying van der Waals equation of state which is used by our LB model to induce phase separation at the interface of the two phases (see Ref. [10] for more details). The kinematic viscosity is taken again equal in both phases, $v_{i}=\frac{1}{6} c \delta x$, where $c=\delta x / \delta t$ is the lattice speed and $\delta t$ is the time step. The interfacial tension is controlled by an adjustable parameter $\kappa$ (as also discussed in Ref. [10]), taken equal to $\kappa=1 \times 10^{-1} c \delta x^{2} / \rho_{0}$, which produces a constant interfacial tension equal to $\gamma=10^{-2} \rho_{0} c^{2} \delta x$.

For each value of $S_{n w}$, we start our simulations with a very dispersed initial size distribution for the ganglia phase pursuing an average size much smaller than the typical pore size. This is achieved by appropriately setting the correlation length values for the solid phase, $\lambda_{s}$, and the nwphase, $\lambda_{n w}$, respectively. This initial condition was selected in order to minimize the capillary effects at early times and allow for the mobilization of the entire ganglia population. Starting from this distribution, the system is simulated over several million time steps, $\delta t$, at a constant Bo value, and it progressively reaches a quasisteady state, where the superficial velocity of both phases, $\hat{u}_{w}$ and $\hat{u}_{n w}$, and the number of ganglia remain practically constant with time. This corresponds to a dynamic equilibrium, where the rate of ganglia coalescence becomes equal to the rate of fragmentation, as already discussed in Ref. [10]. When this condition is fulfilled, a spatially periodic clustering algorithm is applied over all recorded steady-state time steps in order to identify isolated nw-phase clusters (i.e., individual ganglia) and the $x, y$ velocity components of each ganglion are calculated by integrating over their surface. This is discussed in more detail in a subsequent section. We should note here that the clustering algorithm is based on MATLAB's "regionprops" function that measures the structural properties (i.e., number of pixels, center of mass, and acentricity) of isolated ganglia in the phase distribution snapshots produced by our LB code. This is appropriately modified to identify all the clusters of the nw-phase that extend over the sides of our domains and then resolve their periodicity by merging those that appear on both the opposite sides. Once the ganglia velocity has been identified, the ganglia are characterized as immobile, corresponding to the criterion $u_{g}<$ $0.15 u_{0}\left(\mathrm{Bo} ; S_{n w}=1\right)$.

Figure 3 shows three snapshots of the phase distribution patterns under steady-state flow conditions for different values of the applied Bo. The complete videos for these simulations are also provided in the Supplemental Material [49]. We can clearly distinguish significant structural differences for the ganglia populations across these snapshots (and the corresponding videos) as the body force is increased from the lower value of $\mathrm{Bo}=0.01$ shown in Fig. 3(a) towards higher values in Figs. 3(b) and 3(c). These structural changes and their effects on the rheological behavior of the fluids are discussed in the following sections.

\section{A. Effective phase rheology}

We first focus on the superficial two-phase velocity of both phases, $\hat{u}_{t}$, with respect to the applied body force. These are calculated using Eq. (1) averaged over all recorded time steps after the system has reached its "steady state." Figure 4(a) shows the overall Darcy velocity $\hat{u}_{t}=\hat{u}_{n w}+\hat{u}_{w}$ as a function of the applied Bo for different values of the nw-phase saturation, $S_{n w} \leqslant 0.5$, where the nw-phase flows in the form of disconnected ganglia rather than as a percolating phase that spans the 


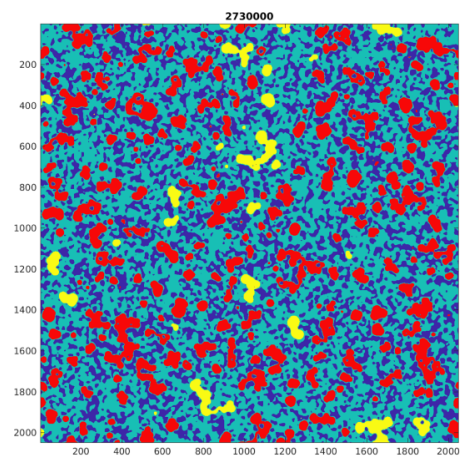

(a)

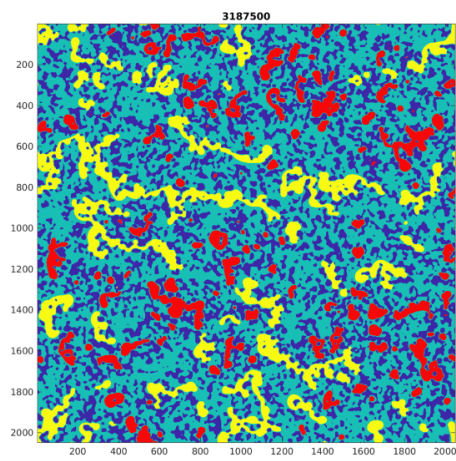

(b)

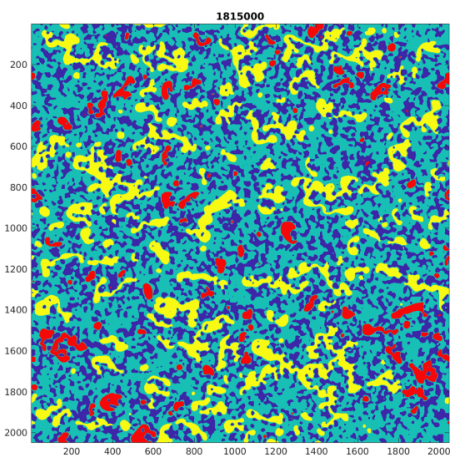

(c)

FIG. 3. Snapshots of ganglia distributions over the entire computational domain at steady-state flow conditions for $S_{n w}=0.3$ and three different values of Bo: (a) Bo $=0.01$, (b) Bo $=0.1$, and (c) Bo $=0.2$. The mobile ganglia are shown in yellow, the stranded ganglia are shown in red, and the solid sites in darker blue color. The complete videos for these simulations are provided in the Supplemental Material [49]. Reported lengths are in lattice space units, $\delta x$, while the simulation time is denoted above each snapshot in $\delta t$ units.

entire domain. Also shown with a dot-dashed line is the corresponding single-phase Darcy velocity scaling, calculated in the limit where $S_{n w}=0$.

Interestingly enough, the presence of the nw-phase in the domain leads to a significant decrease of $\hat{u}_{t}$ over the entire range of Bo values and a divergence from the reference single-phase curve which becomes particularly apparent for larger values of $S_{n w}$ and lower values of Bo. Furthermore, the two-phase velocity curves are found to exhibit three distinct flow regimes: (a) a low-Bo regime, where $\hat{u}_{t}$ scales as $\hat{u}_{t} \propto B o$ in a similar fashion as the reference single-phase curve, but exhibiting a monotonically decreasing relative permeability for increasing values of $S_{n w}$ (as denoted by the intercept of each curve with the velocity axis), (b) an intermediate-Bo regime, where a saturation-dependent nonlinear Darcian scaling is evident, i.e., $\hat{u}_{t} \propto \mathrm{Bo}^{a}$ with $a=a\left(S_{n w}\right)>1$, and (c) a high-Bo regime, where the linear Darcian scaling, $\hat{u}_{t} \propto$ Bo, is progressively restored, reaching

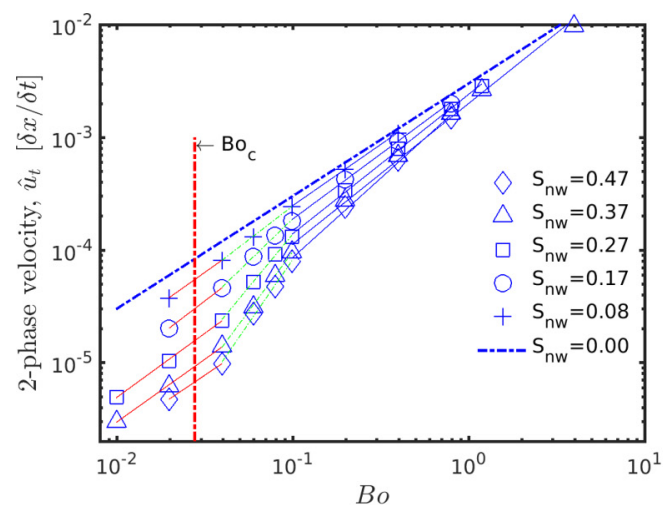

(a)

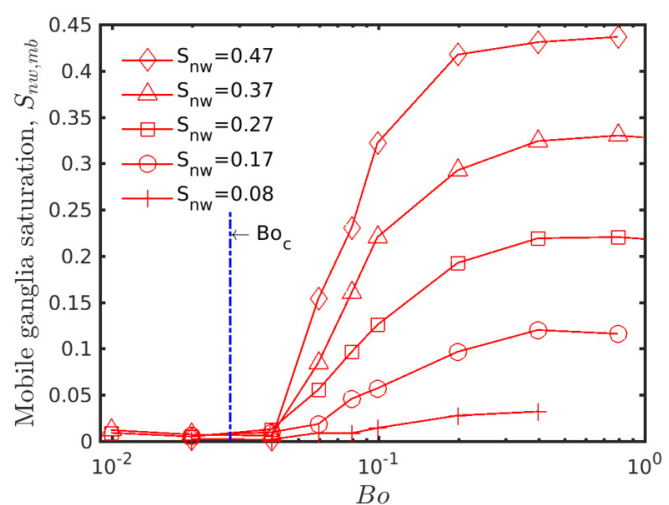

(b)

FIG. 4. (a) Superficial (Darcy) two-phase velocity, $\hat{u}_{t}$, scaling with applied Bo for various values of the nw-phase saturation, $S_{n w}$. The dot-dashed line corresponds to our reference single-phase flow scaling, namely, for $S_{n w}=0$. (b) Mobile nw-phase saturation, $S_{n w, m b}$, as a function of applied Bo. Note that at high Bo values all curves reach asymptotically a plateau, $S_{n w, m b}=S_{n w}-S_{n w, r}$, which corresponds to a fixed residual nw-phase saturation value of $S_{n w, r} \approx 0.05$. 
asymptotically the single-phase reference curve, namely, the relative permeability reaches unity regardless of the value of $S_{n w}$.

A similar sequence of scalings has also been reported both experimentally and numerically in recent literature [15,27]. Our simulations, however, reveal an additional dependence of the scaling exponent $a$ on the nw-phase saturation, $S_{n w}$, which has not been reported in the literature.

A straightforward reasoning for the above behavior becomes apparent when we plot the mobile nw-phase saturation, $S_{n w, m b}$ (namely, the overall saturation corresponding to the ganglia that have been identified as mobile) vs Bo, shown in Fig. 4(b). This reveals that within the low-Bo regime all ganglia remain stranded (i.e., $S_{n w, m b} \approx 0$ ), thus blocking some of the available flow paths and decreasing the effective permeability of the medium. It is in this region that the most important divergence from the reference single-phase velocity is observed. Therefore, this particular regime can be viewed as a single-phase flow process where the relative permeability of the wetting phase is a decreasing function of $S_{n w}$, as revealed by the ever decreasing curve intercept with the $\hat{u}_{t}$ axis.

As Bo increases above a critical value $\mathrm{Bo}_{c}$ (which appears to be independent of $S_{n w}$ in our study, but could depend on the characteristics of the medium), the mobile ganglia saturation increases, abruptly reaching asymptotically the value $S_{n w}-S_{n w, r}$, where $S_{n w, r}$ is the residual (irreducible) nw-phase saturation. It is interesting to note that, despite the stochastic nature of all our domains, $S_{n w, r}$ is found approximately equal to 0.05 in all our simulations, and independent of the value of $S_{n w}$. This fact implies that $S_{n w, r}$ may depend solely on the geometrical characteristics of the domain, i.e., the porosity, solid-phase correlation length, and its autocorrelation function.

The intermediate-Bo regime thus exhibits a scaling exponent larger than unity due to the gradual mobilization of ganglia and the subsequent opening of new flow paths. Finally, the high-Ca regime corresponds to a viscosity-dominated flow, where the energy dissipated due to capillarity effects is negligible. Therefore, the velocity scaling gradually converges towards the reference single-phase flow curve. An interesting observation is also that the residual saturation (due to stranded ganglia), $S_{n w, r}$, does not significantly alter the effective permeability of the domains (as it happens in the lowBo regime) given that the residual ganglia are expected to be small and stranded in flow stagnation regions that have a negligible contribution to the effective flow paths.

In order to further analyze the physical origins of the above scalings, we study the distinct behavior of each of the two phases, separately. Starting from the w-phase, we observe in Fig. 5(a) that it exhibits a practically linear scaling with Bo regardless of $S_{n w}$. In fact, the whole flow rate curve could be fitted with a power law with an exponent slightly different than 1 , although the higher Bo data points clearly exhibit the expected linear behavior. This could suggest that the w-phase curve also undergoes a change of behavior depending on the value of Bo, but this would fall below the statistical error in our simulation. Moreover, it is evident that the relative permeability of the w-phase is a decreasing function of $S_{n w}$ regardless of the ratio of stranded to total nw-phase saturation.

The nw-phase [shown in Fig. 5(b)], however, exhibits a more complex behavior which is consistent with the two-phase velocity scaling. Here, again we can distinguish three regimes. At low Bo, the nw-phase velocity is practically equal to zero and does not appear in this logarithmic plot. Above a certain critical Bond number $\mathrm{Bo}_{c}$, the nw-phase starts to flow with a flow rate increasing faster than linear growth. Finally, for larger Bo values the flow rate recovers a linear relationship. Interestingly, we find that all the data points from all our simulations collapse to a single curve using the following scaling laws: $\hat{u}_{n w} \propto \frac{k}{v}\left(S_{n w}-S_{n w, r}\right) f\left(\left|\mathrm{Bo}-\mathrm{Bo}_{c}\right|\right)$, where $S_{n w, r} \approx 0.05$ is the residual saturation, $\mathrm{Bo}_{c} \approx 3 \times 10^{-2}$, and $f(x)$ is a function that undergoes a change of power-law scalings with an exponent equal to $3 / 2$ when $x$ is small and an exponent of 1 for large values of $x$. This law also expresses the fact that below $\mathrm{Bo}<\mathrm{Bo}_{c}$ (or $S<S_{r}$ ), the nonwetting phase is not flowing. It also suggests that at a given $\mathrm{Bo}$, the relative permeability is linear with saturation.

\section{B. Single- and two-phase flow paths}

We proceed with the study of the statistical properties of the flow field in order to shed light upon the pore-scale origins of the nonlinear scaling regime observed both for the total and the 


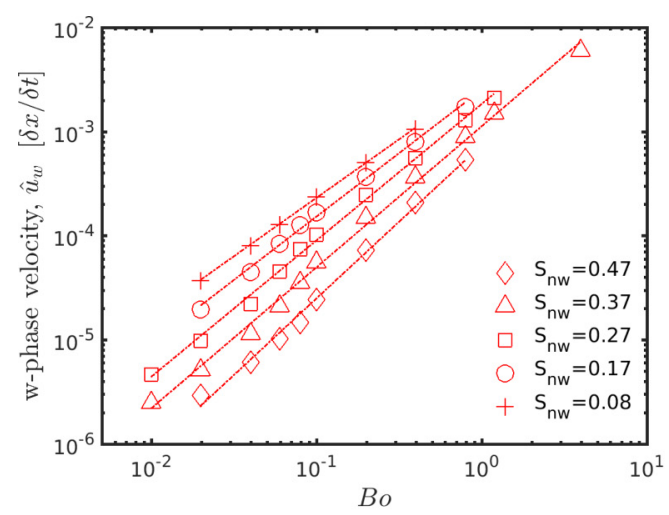

(a)

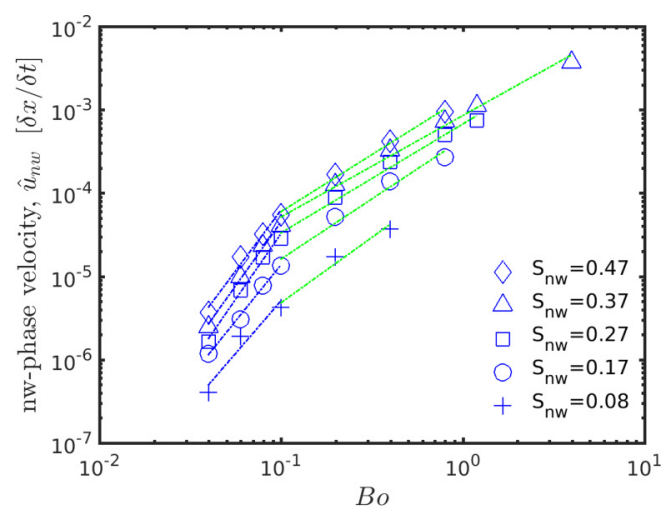

(b)

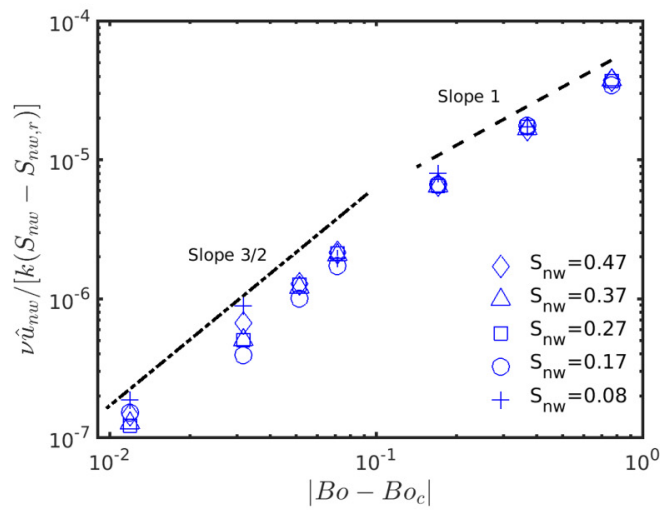

(c)

FIG. 5. Superficial (Darcy) velocity scaling with Bo (a) for the w-phase and (b) for the nw-phase (ganglia). (c) The rescaled nw-phase velocity with respect to $\left|\mathrm{Bo}-\mathrm{Bo}_{c}\right|$.

nw-phase velocities in the intermediate-Bo region. As discussed in the Introduction and also indirectly observed in Fig. 4(b), it was hypothesized that this regime may be related to the gradual mobilization of ganglia that results in the "opening" of new flow paths, in a similar fashion as the nonlinear flow of yield-stress fluids in porous media near critical pressure. We postulate that the pore-scale disorder generates two-phase flow paths with different characteristic (average) hydraulic diameters normal to the dominant flow direction. This variance of flow path diameters results in a nonlinear increase of the flow rate with the imposed pressure drop (or Bo) as viscous (or gravity) forces gradually overcome capillary ones (the latter being inversely proportional to the characteristic flow path diameter).

To verify this assumption, we define a nw-phase residence function, $P(x, y, t)$, as being equal to unity for sites occupied by the nw-phase, and equal to zero, otherwise. We can therefore define the spatial probability function $p(x, y)=\langle P(x, y, t)\rangle_{t}$, which is averaged over the entire range of the recorded "steady-state" time steps for each realization. Figure 6 shows the nw-phase residence probability function $p(x, y)$ for a single reconstructed domain and different Bo values in the intermediate-Bo regime. For the lower Bo value [shown in Fig. 6(a)], the nw-phase is mainly immobile, which is characterized by $p(x, y)$ being equal to either zero (always w-phase or solid sites) or unity (always nw-phase). As Bo increases in Figs. 6(b) and 6(c), $p(x, y)$ takes intermediate values corresponding to regions alternating between the $\mathrm{w}$ - and nw-phases, due to the progressive mobilization of ganglia. Some preferential flow paths can be distinguished using this approach. 


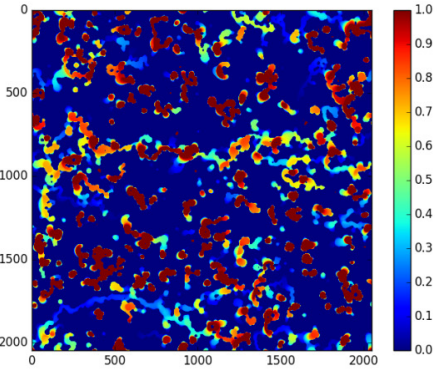

(a)

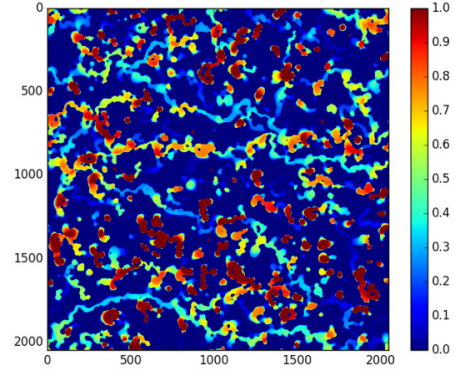

(b)

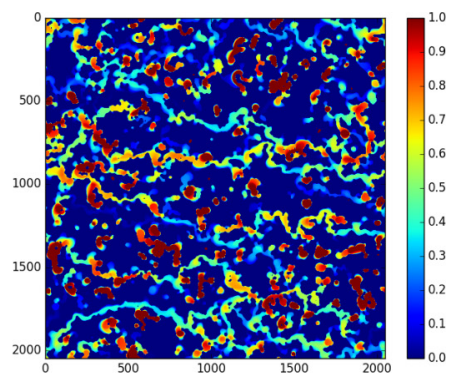

(c)

FIG. 6. Mean presence maps $p(x, y)$ of ganglia saturation (nw-phase) over all recorded time steps at steadystate conditions for $S_{n w}=0.3$ and (a) Bo $=0.06$, (b) Bo $=0.08$, and (c) Bo $=0.1$. All lengths are in lattice space units, $\delta x$.

The flow paths are even more evident when we plot the nw-phase probability velocity, defined as the steady-state temporally averaged local velocity multiplied by nw-phase presence function, $C(x, y)=\left\langle P(x, y, t) \times u_{n w}(x, y, t)\right\rangle_{t}$. Using this approach, we can eliminate the velocity of the wphase, as well as the stranded ganglia (but not entirely because there is always a small recirculation flow within each stranded ganglion). Figure 7 shows this correlation map for the same Bo values as in Fig. 6 (i.e., in the intermediate-Bo regime where the nonlinear Darcian scaling was previously reported). For all Bo values, several nw-phase flow paths percolating from one side to the other (or the top and the bottom) of the periodic domain can be clearly distinguished. We can also observe that the number of these flow paths is increasing with Bo in a similar fashion to the yield-stress fluid problem, as initially hypothesized. In this case, however, pore throats are invaded when the body force exceeds the local capillary pressure and therefore ganglia become mobilized. In addition, this condition must be met along an entire percolating path; otherwise, the ganglion will become once again stranded after a transitional time of short-term mobility. As Bo (or the pressure drop) increases, more flow paths meet this condition, resulting in an apparent increase in the relative permeability of the medium. In our simulations, this is manifested by the power-law scaling of the total and the nw-phase velocity with Bo, as discussed in the previous sections.

If we want to schematize this flow problem, one can distinguish two types of regions in Fig. 6. The first one consists of channels where two-phase flow takes place and whose number depends strongly on the applied Bo. In between these channels, there are also regions composed of the immobile ganglia with the w-phase flowing around them. Therefore, the w-phase flows in both

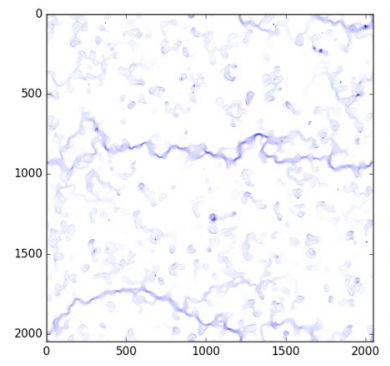

(a)

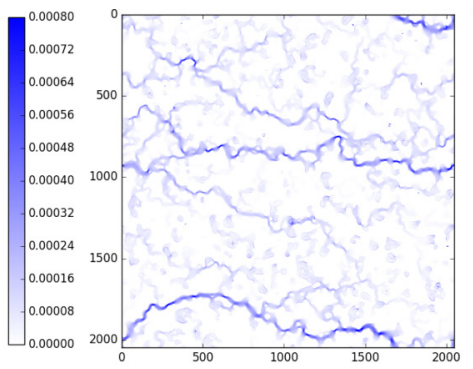

(b)
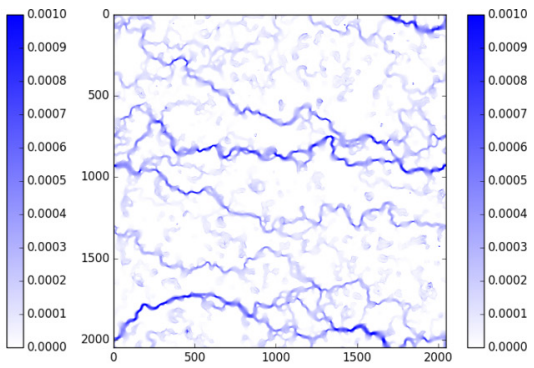

(c)

FIG. 7. Mean correlation maps of nw-phase flux, $C(x, y)=\left\langle P(x, y, t) \times u_{n w}(x, y, t)\right\rangle_{t}$, over all recorded time steps at steady-state conditions for $S_{n w}=0.3$ and (a) Bo $=0.06$, (b) Bo $=0.08$, and (c) Bo $=0.1$. All lengths are in lattice space units, $\delta x$. 


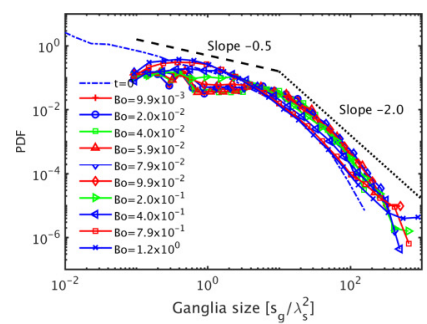

(a)

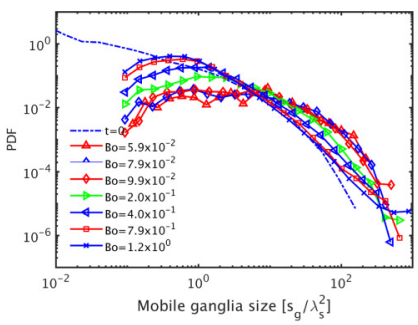

(b)

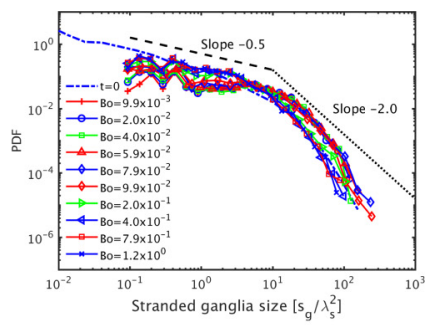

(c)

FIG. 8. Probability density functions of the (a) total, (b) mobile, and (c) stranded ganglia populations for $S_{n w}=0.30$ and different values of Bo. Also shown is the initial ganglia size distribution at $t=0$, and the curves corresponding to the power-law scalings with an exponent of -0.5 (dashed line) and -2 (dotted line).

regions and the analogy with yield-stress fluids should be only applied to the nw-phase liquid. This may explain why the partial flow scaling law in Fig. 5 is more obvious for the nw- than the w-phase. In addition, it should also be noted that the immobile nw-phase regions consist mainly of immobile fluid that has almost never moved. Hence, its configuration depends mostly on the initial state (or history) of nw-phase distribution. For this reason, trying to determine a universal behavior does not seem relevant in our simulations.

\section{Ganglia morphology and size and length probability distributions}

A distinct feature of our numerical approach lies in its ability to identify individual ganglia and classify them as mobile or stranded based on a preselected mobility criterion. We identify individual ganglia at each recorded time step by applying a periodic Hoshen-Kopelman algorithm [50] on the calculated digital phase distribution domains. This algorithm identifies neighboring nw-phase pixels (i.e., lattice voxels occupied by the nw-phase at the current $t$ ) and marks their domain coordinates in order to treat them as a single entity. Given the number of pixels and their coordinates for each ganglion, it is straightforward to calculate its velocity and size by integrating as follows:

$$
u_{x, g}=\frac{1}{s_{g}} \int_{s_{g}} u_{x} d s \text { and } u_{y, g}=\frac{1}{s_{g}} \int_{s_{g}} u_{y} d s,
$$

where $u_{x}, u_{y}$ are the calculated velocity components at each voxel $(x, y)$, and $s_{g}$ is the ganglion size in $\delta x^{2}$ units calculated as the total number of neighboring voxels belonging to each individual ganglion [i.e., local phase index (or density) equal to $\rho_{n w}$ ]. The velocity magnitude of each ganglion is then calculated as $u_{g}=\left(u_{x, g}^{2}+u_{y, g}^{2}\right)^{0.5}$ and the ganglia are subsequently characterized as mobile when their velocity magnitude is at least $15 \%$ that of the single-phase interstitial velocity for the same applied body force, i.e., $u_{g} \geqslant 0.15 u_{n w}\left(\mathrm{Bo} ; S_{n w}=1\right)$. The latter interstitial nw-phase velocity, $u_{n w}$, is calculated based on a single-phase flow realization using only the nw-phase fluid, i.e., by setting $S_{n w}=1$.

Based on the size and mobility of each ganglion, we plot in Fig. 8 the probability density functions (PDFs) for the entire population [Fig. 8(a)], and separately for the mobile [Fig. 8(b)] and stranded [Fig. 8(c)] ganglia. Note that the ganglion size, $s_{g}$, in these plots has been normalized with $\lambda_{s}^{2}$, which denotes the typical pore size in our domains. Figure 8 shows the results obtained over all domain realizations at all recorded steady-state time steps and reveals some distinct characteristics of the two populations that to the knowledge of the authors have never been reported before. The PDF of the stranded population is reminiscent of those reported in earlier experimental works (e.g., Ref. [14]), which is characterized by a smaller-than-average pore size scaling of $p(s) \propto s^{-0.5}$ and a larger-than-average pore size scaling of $p(s) \propto s^{-2}$. These two scalings are also plotted in the stranded and total ganglia population PDFs with dashed and dotted lines, respectively, along with the initial ganglia size PDF (i.e., at $t=0$ ) that appears to follow a $\Gamma$ probability distribution. Although 


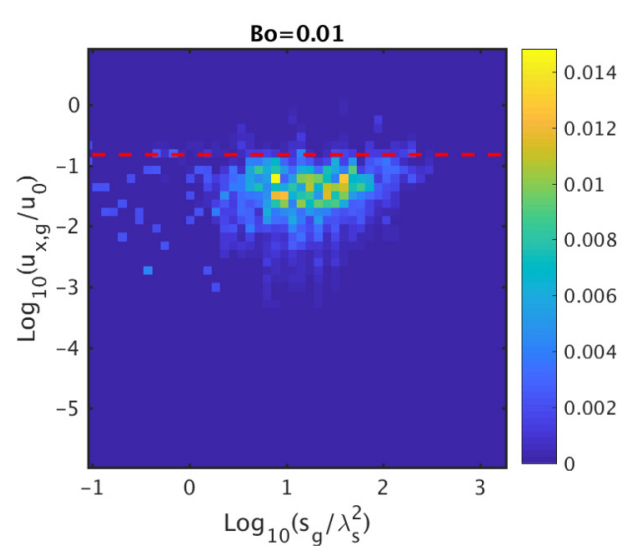

(a)

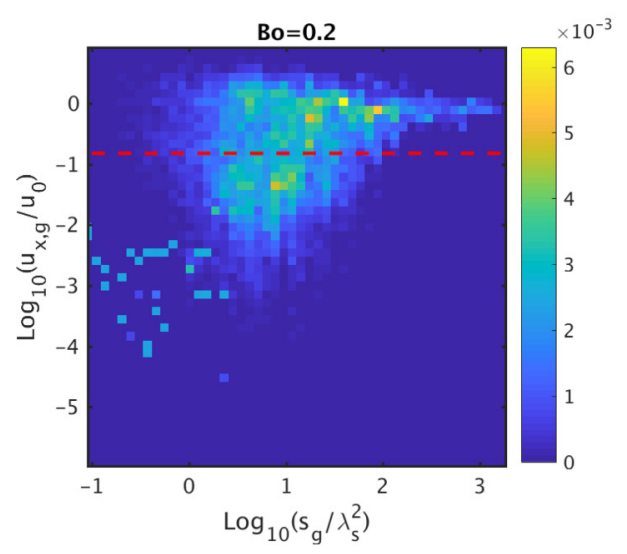

(c)

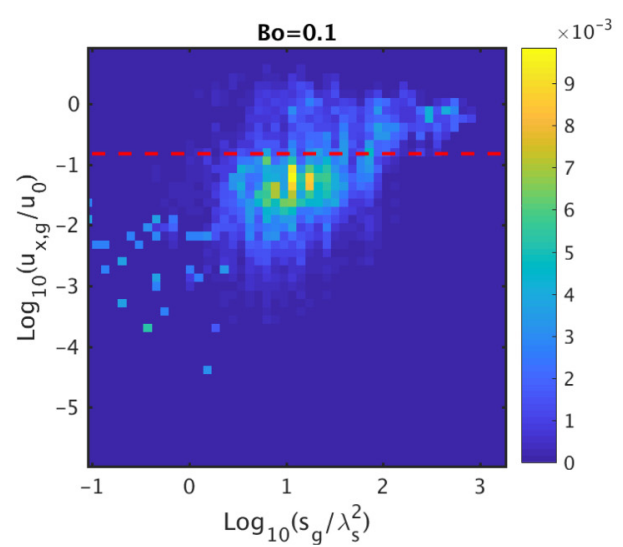

(b)

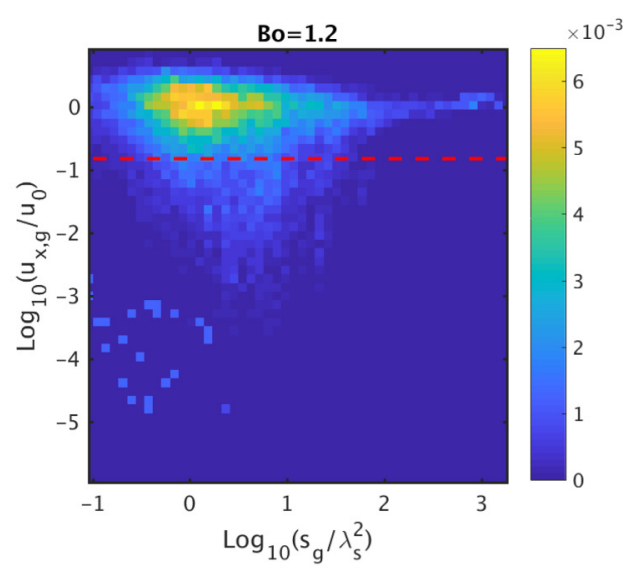

(d)

FIG. 9. Probability density maps of ganglia velocity in the flow direction, $u_{x, g} / u_{0}$, and size, $s_{g} / \lambda_{s}^{2}$, over two orders of magnitude of Bo for $S_{n w}=0.4$. The dashed red line denotes the applied threshold criterion between mobile and stranded ganglia and corresponds to a relative permeability of 0.15 .

our results are not conclusive regarding these scalings, there also appears to be a non-negligible dependence of these curves on Bo, which should be further investigated.

The behavior, however, of the mobile ganglia population is clearly strongly dependent on Bo and the curves are qualitatively different from previously published results. Our results show a clear shift of the mobile population towards larger sizes as Bo decreases, while smaller sizes are dominant at larger Bo values. This should be attributed to the pore-scale physics related to ganglia mobilization. As also discussed in the previous sections, larger ganglia become mobilized at smaller Bo due to the larger pressure difference (or overall larger body force) across their length in the flow direction. Therefore, at lower Bo values in the intermediate regime, flow is dominated by larger ganglia while those of size comparable to the typical pore size remain stranded. As Bo increases, viscous (or gravity) forces dominate over capillarity leading to the subsequent mobilization of smaller ones, but also to the intensification of the dynamic fragmentation process. This results in the shifting of the curves towards smaller sizes, while the large ones become more rare.

The distinct characteristics of the two ganglia populations are further investigated in the bivariate frequency maps shown in Fig. 9. Here we plot the combined occurrence probability of ganglia with 
$u_{x, g} / u_{0}$ velocity in the flow direction and size equal to $s_{g} / \lambda_{s}^{2}$, where $u_{0}=u_{n w}\left(\mathrm{Bo} ; S_{n w}=1\right)$ stands for the average interstitial velocity of the nw-phase at the same Bo for $S_{n w}=1$. The plots cover the entire population of mobile and stranded ganglia and include all three domain realizations and all time steps recorded at steady state. We have also plotted with a red dashed line the threshold velocity above which the ganglia are characterized as mobile, corresponding to the criterion $u_{g} \geqslant$ $0.15 u_{0}\left(\mathrm{Bo} ; S_{n w}=1\right)$.

Starting from the lower value of $\mathrm{Bo}=0.01$ [Fig. 9(a)], which was selected to be below $\mathrm{Bo}_{c}$, it is clear that practically the entire population lies below the selected velocity threshold, regardless of size, and in a velocity region between $0.1 u_{0}$ and $0.01 u_{0}$. Furthermore, it appears to follow a Gaussian size distribution with a mean in the region $10 \lambda_{s}^{2}-100 \lambda_{s}^{2}$. Given that all ganglia are macroscopically "stranded," these nonzero velocities should be attributed to an internal circulation inside them due to the viscous shear stresses imposed across the interfaces by the flowing w-phase.

An increase of Bo by an order of magnitude [i.e., Bo $=0.1$ in Fig. 9(b)] leads to the mobilization of larger ganglia, demonstrated as a "jump" in velocity and repositioning in the mobile region with a typical velocity on the order of $0.5 u_{0}-1.0 u_{0}$. The majority, however, of the population still remains in the stranded region as denoted by the relevant intensity colors.

A further increase of Bo leads to the transition of the majority of the population in the mobile region [as more clearly shown for Bo $=1.2$ in Fig. 9(d)], while the most probable size now becomes much smaller and on the order of $\lambda_{s}^{2}-10 \lambda_{s}^{2}$. The average ganglia velocity is now on the order of magnitude of $u_{0}$. This analysis further highlights the different size probability distributions of the two populations, discussed also earlier, and verifies the proper choice of the velocity threshold to distinguish between mobile and stranded ganglia.

We should also note here the presence of a very distinct population of stranded ganglia with persistent and very small sizes, $20 \delta x^{2} \geqslant s_{g} \geqslant 230 \delta x^{2}$, over the entire duration of our numerical simulations. This population appears practically in all our simulations as scattered discontinuous points in Figs. 9(a)-9(d) in the lower left region of the plots. We believe that this population is associated with the residual saturation, $S_{n w, r}$, and their persistent size is determined by the specific structural characteristics of the three reconstructed porous domains.

Figure 10 shows the orientation of individual ganglia, $\theta=\tan ^{-1}\left(u_{y, g} / u_{x, g}\right)$, with respect to the dominant flow direction for $S_{n w}=0.4$ and increasing values of Bo. The selected Bo values match exactly the ones of Fig. 9. Starting from the lower value of $\mathrm{Bo}=10^{-2}$ in Fig. 10(a), where we have seen previously that all ganglia are stranded, we note a practically uniform orientation distribution of the ganglia regardless of their size, which ranges on the order of $\lambda_{s}^{2}-10 \lambda_{s}^{2}$. Obviously, this corresponds to a local movement of the ganglia within the pore space, where they remain stranded. As Bo increases, the larger ganglia become mobilized and align their movement along the dominant flow direction. It is also interesting to note that the distribution is symmetric around $\theta=0$, revealing once again the isotropic nature of our digital domains. For Bo $=1.2$ in Fig. 10(d), the ganglia distribution becomes even wider, spanning from sizes on the order of $0.2 \lambda_{s}^{2}$ to $10^{3} \lambda_{s}^{2}$ (i.e., more than three orders of magnitude). The orientation of smaller ones in the range $\lambda_{s}^{2}-10 \lambda_{s}^{2}$ appears to follow a Gaussian distribution, while the larger ones are practically oriented along the dominant flow direction.

We conclude this section by discussing the length of the ganglia in relation to their size. For this purpose, we used MATLAB to fit an ellipse over each ganglion that has the same normalized second central moments. The calculated major and minor axes of the fitted ellipse correspond to the length and width of the ganglion, $l_{\max }$ and $l_{\min }$, respectively. Figure 11(a) shows the average ganglion length $l_{\max }$ and width $l_{\min }$ with respect to their size $s_{g}$, that clearly reveals an interesting structural change around $s_{g} \simeq \lambda_{s}^{2}$, which is reminiscent of the one also observed in the size PDFs. Indeed, for smaller sizes, both lengths $\left(l_{\max }\right.$ and $\left.l_{\min }\right)$ seem to scale with ganglia size as $l_{\max } \sim l_{\min } \propto s^{1 / 2}$, which indicates that smaller ganglia have an invariant, almost isotropic shape. For larger sizes, however, while both lengths still follow a power law with size, the exponent becomes quite different: $l_{\max } \propto s^{0.72}$ for the ganglion length, and $l_{\min } \propto s^{0.5}$ for the ganglion width. This indicates a self-affine 


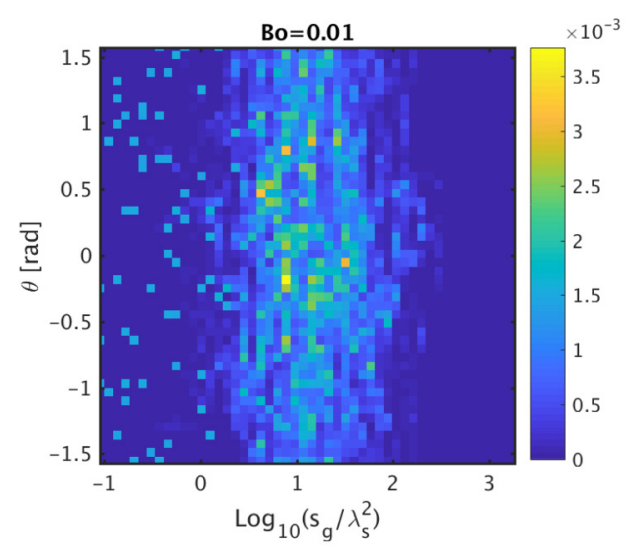

(a)

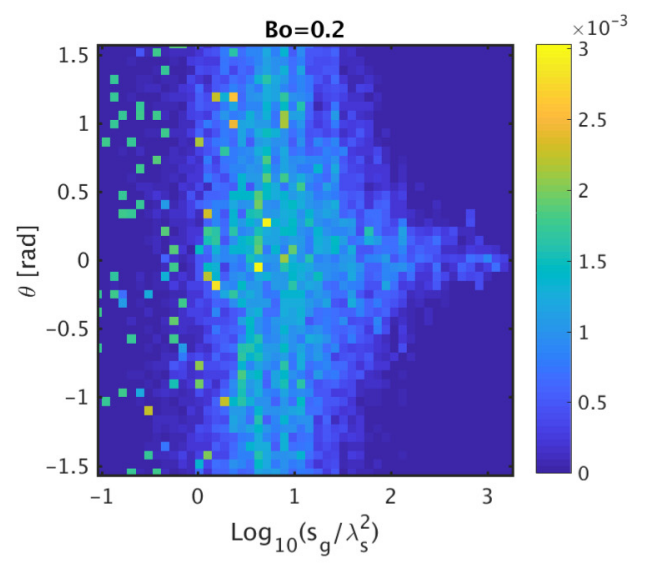

(c)

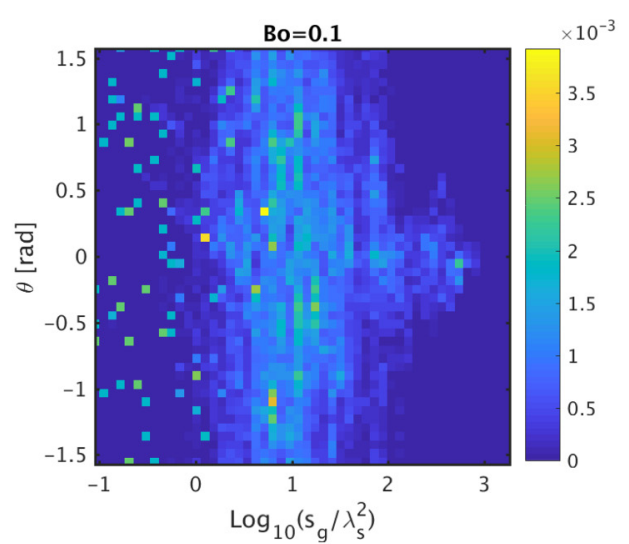

(b)

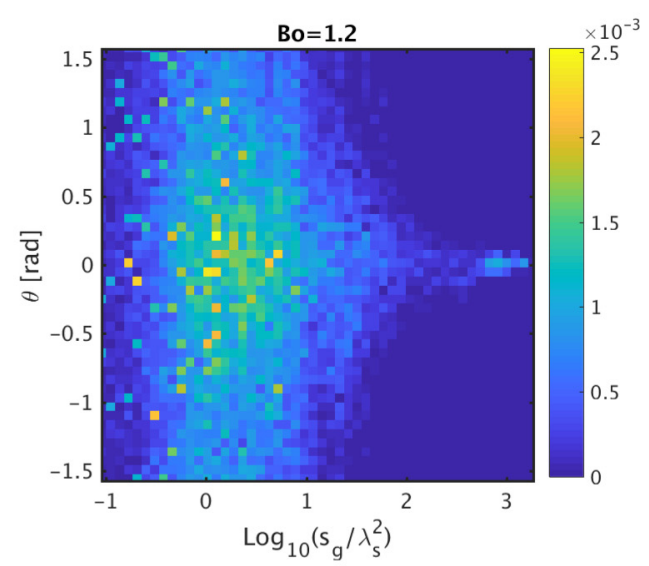

(d)

FIG. 10. Probability density maps of ganglia flow direction with respect to the applied body force, $\theta$, and size, $s_{g} / \lambda_{s}^{2}$, over two orders of magnitude of Bo for $S_{n w}=0.4$. The scattered points in the left side of these maps should be attributed to very small stranded ganglia with sizes determined by the specific structural properties of our reconstructed domains. These constitute (at least partly) the reported residual saturation, $S_{n w, r}$.

property. The fact that $l_{\max }$ increases faster than $l_{\min }$ confirms the visual observation that larger ganglia are much more elongated than smaller ones (see also Fig. 3). In addition, it should be noted that the average size is proportional to the product $l_{\max } l_{\min } \propto s^{1.22}$, as shown in Fig. 11(b), which would indicate that larger ganglia exhibit a fractal character.

It is interesting to compare these results with the work of Tallakstad et al. [11], who performed two-phase flow experiments within monolayer bead packings. Contrary to what is observed here, they reported similar exponents for both the length and width of ganglia, i.e., $l_{\max } \propto s^{0.55}$ and $l_{\min } \propto s^{0.55}$, regardless of size. This apparent difference could be attributed to the large porosity of our domains which allows for the formation of smaller-than-average pore size ganglia, while, in the work of Tallakstad et al., even the smaller ganglia appear to cover several pore bodies. Another contributing factor to this discrepancy could be related to the finite depth of the cells in these experiments (compared to the infinite depth of our 2D simulations), which could further intensify capillary effects, thus leading to more isotropic ganglia over the entire range of sizes. 


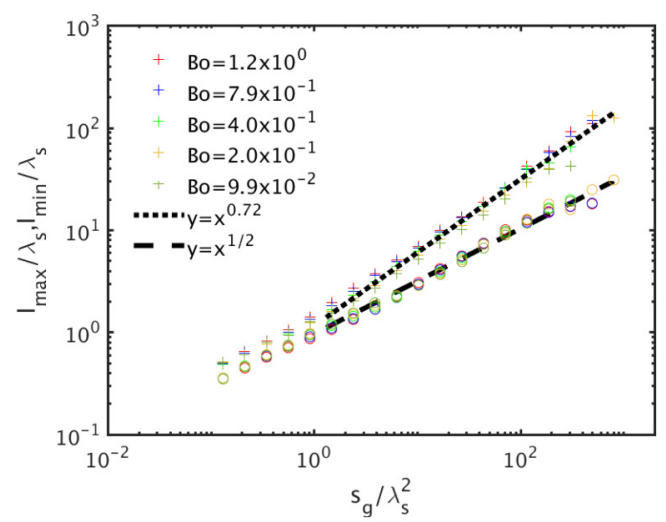

(a)

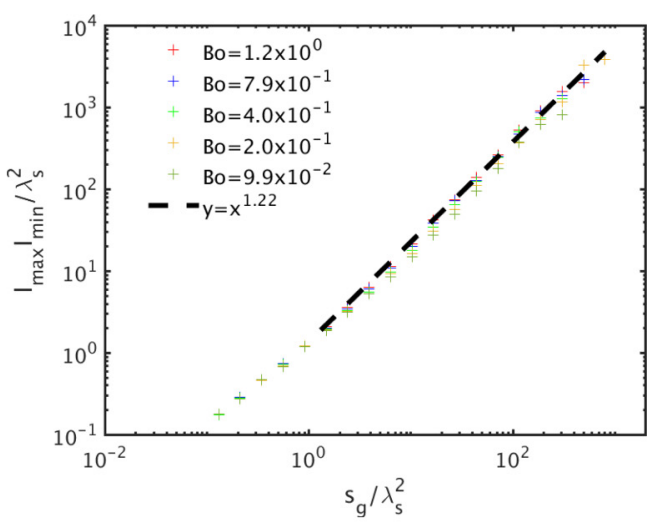

(b)

FIG. 11. (a) Mean ganglion length $\left(l_{\max }\right.$, crosses) and width $\left(l_{\min }\right.$, circles) as a function of size, $s_{g} / \lambda_{s}^{2}$, for different Bo values. The dashed line represents a power law of $s_{g}^{0.5}$ and the dotted line represents $s_{g}^{0.72}$. (b) Product of mean ganglion length and width as a function of size. The dashed line represents $s_{g}^{1.22}$.

\section{Flow velocity of the w-phase}

We conclude our study with a discussion on the effects of the presence of ganglia on the w-phase velocity distribution. As we discussed in the previous sections, in the low-Bo limit (i.e.,Bo $\leqslant \mathrm{Bo}_{c}$ ) all ganglia remain practically stranded and the system undergoes only single-phase flow of the wphase. The presence of the stranded ganglia affects the relative permeability of the w-phase (which was found to be a monotonically decreasing function of $S_{n w}$; see, e.g., Fig. 5) by blocking the available flow paths (as revealed in Fig. 7). This is also expected to have a non-negligible effect on the distribution of the $\mathrm{w}$-phase velocities at the pore scale.

We thus define the local w-phase velocity as $\vec{u}_{w}(x, y)$, with components $u_{x}(x, y)=\vec{n} \cdot \vec{u}_{w}$ and $u_{y}(x, y)=\vec{t} \cdot \vec{u}_{w}$, where $\vec{n}$ and $\vec{t}$ are the unit vectors in the longitudinal and transverse direction of the applied $\vec{F}$, respectively. Figure 12 shows the PDF of the longitudinal, $u_{x}(x, y) /\left(\hat{u}_{w} /\left(\phi S_{w}\right)\right)$, and transverse velocity components, $u_{y}(x, y) /\left(\hat{u}_{w} /\left(\phi S_{w}\right)\right)$, of the w-phase over all domain realizations and averaged over all recorded steady-state time steps for $\mathrm{Bo} \approx \mathrm{Bo}_{c}=3 \times 10^{-2}$. Here the velocity components are rescaled with the average w-phase interstitial velocity, $\hat{u}_{w} /\left(\phi S_{w}\right)$. In the absence of ganglia $\left(S_{n w}=0\right)$, Fig. 12 reveals a remarkable exponential probability distribution for both velocity components. For the transverse component, the PDF is found to be symmetric around $u_{y}=0$, as expected, spanning over several orders of magnitude in probability. For the longitudinal

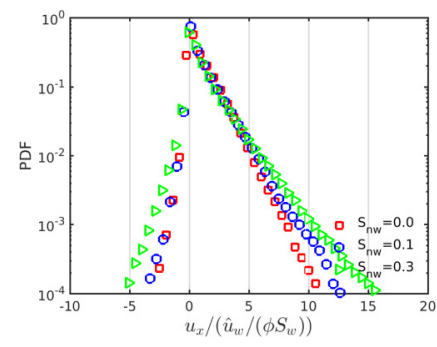

(a)

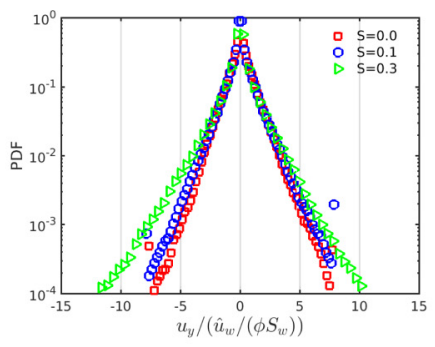

(b)

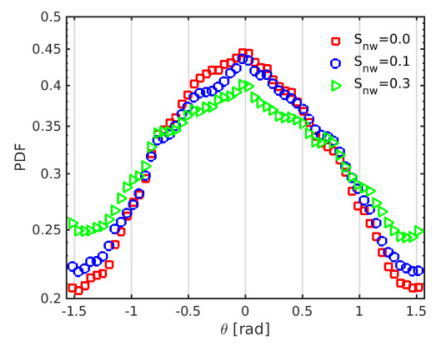

(c)

FIG. 12. Probability density functions (PDFs) of the rescaled (a) longitudinal, $u_{x}$, and (b) transverse velocity, $u_{y}$, components, and (c) orientation, $\theta=\tan ^{-1}\left(u_{y} / u_{x}\right)$, of the w-phase velocity vector $\vec{u}_{w}$ for various values of the nw-phase saturation in the limit where all ganglia are immobile (i.e., $\mathrm{Bo} \leqslant \mathrm{Bo}_{c}$ ). 
velocity components $u_{x}$, the PDF is not symmetric, but rather biased towards the positive velocity components in the flow direction, as intuitively expected. Only the largest longitudinal components seem to follow an exponential decay.

The presence, however, of the stranded ganglia for $S_{n w}=0.1$ and 0.3 appears to increase the tails of both distributions, indicating an increase of probability for larger velocity magnitudes, compared to an actual single-phase simulation (i.e., $S_{n w}=0$ ). These changes should be attributed to the decrease in the pore space available for the flow of the nw-phase (for increasing $S_{n w}$ ) combined with the "slip" (lubricating) effects at the fluid-fluid interfaces. Both these effects lead to increased interstitial velocities for the w-phase.

Also shown in Fig. 12 is the w-phase flow orientation, $\theta=\tan ^{-1}\left(u_{y} / u_{x}\right)$, that reveals a broad but symmetric distribution around $\theta=0$ (i.e., the principal flow direction) and exhibits again a nearly exponential distribution. This demonstrates the isotropic and homogeneous character of the medium on a length scale equal to the digital domain size. The presence of stranded ganglia once again acts in the direction of "smoothing" the slopes towards a more uniform distribution for the velocity orientation. These results are in excellent qualitative agreement with the experimental data of Datta et al. [51] obtained using confocal microscopy in 3D sintered glass bead packings. This demonstrates that the above reported exponential distributions are independent of the dimensionality of the system.

\section{CONCLUSIONS}

In this contribution, we studied the flow of immiscible nonwetting liquid ganglia in heterogeneous 2D porous structures using an elaborate pore-scale lattice Boltzmann simulator. In line with earlier works, we observed the existence of a nonlinear Darcy-scale regime where the total flow rate follows a power-law scaling with applied Bo. We found, however, that the scaling exponent is a strong function of the nw-phase saturation, contrary to the conjecture of a universal exponent proposed by Sinha and Hansen [27]. Considering each phase separately, we showed that the nw-phase superficial velocity also exhibits a power-law regime with an exponent equal to $3 / 2$ and independent of $S_{n w}$, while the w-phase appears to follow a practically linear scaling with Bo. By analogy with the problem of yield-stress fluids in porous media [52], we attributed the emergence of such a regime to the presence of spatial heterogeneities that lead to the development of preferential flow paths for the nw-phase. Our simulations revealed that the number of such flow paths depends strongly on Bo, thus leading to this nonlinear Darcy-scale behavior.

The above transitional regime is observed when the applied Bo increases above a critical value, which was found in our study to be equal to $\mathrm{Bo}_{\mathrm{c}} \approx 3 \times 10^{-2}$, and independent of $S_{n w}$. As shown in the supplemental videos [49], this value marks the transition from single-phase flow of the w-phase around the entire population of stranded ganglia to the percolating mobilization of the first (larger) ganglion and the creation of the first two-phase flow path. We expect that, assuming a very dispersed initial ganglia distribution (as in this study), this critical value is a characteristic of the geometrical (pore-scale) properties of the porous domain, including its dimensionality, pore connectivity, and variance of the size distribution of the solid obstacles. We would also expect that a large variance in the pore size distribution would result in a more extended nonlinear two-phase flow regime that could well span over several orders of magnitude of Bo.

We also studied the rheological and structural characteristics of ganglia with respect to $S_{n w}$ and Bo. The size distribution of the stranded ganglia was found to exhibit two distinct power-law scalings at small and larger sizes, which were, however, insensitive to Bo. On the other hand, the size distribution of the mobile ganglia was found to evolve significantly with Bo with a characteristic shift towards smaller sizes for increasing Bo values. Furthermore, the shape of smaller ganglia was found to be practically isotropic, while larger ganglia exhibit a self-affine property, where their width and length both follow a power-law dependency with size, but with different exponents. 


\section{ACKNOWLEDGMENTS}

The authors acknowledge the generous allocation of computational resources required for this study by the Greek Research \& Technology Network (GRNET) on the National HPC facility ARIS (http://hpc.grnet.gr) under projects LB-BLOB and LB-BLOB2. The work of A.Y. and A.D. has been supported by the Greek Secretariat for Research and Technology (GSRT) and the Hellenic Foundation for Research and Innovation (HFRI) through the research grant "3DmicroFlows." This work was also supported by the "ADI 2018" project funded by the IDEX Paris-Saclay, ANR-11IDEX-0003-02.

[1] F. M. Orr and J. J. Taber, Use of carbon-dioxide in enhanced oil recovery, Science 224, 563 (1984).

[2] J. E. McGray, G. R. Tick, J. W. Jawitz, J. S. Gierke, M. L. Brusseau, R. W. Falta, R. C. Knox, D. A. Sabatini, M. D. Annable, J. H. Harwell, and A. L. Wood, Remediation of NAPL source zones: Lessons learned from field studies at Hill and Dover AFB, Ground Water 49, 727 (2011).

[3] S. Bachu and J. J. Adams, Sequestration of $\mathrm{CO}_{2}$ in geological media in response to climate change: Capacity of deep saline aquifers to sequester $\mathrm{CO}_{2}$ in solution, Energy Convers. Manage. 44, 3151 (2003).

[4] D. G. Avraam and A. C. Payatakes, Generalized relative permeability coefficients during steady-state 2-phase flow in porous media, and correlation with the flow mechanisms, Transp. Porous Media 20, 135 (1995).

[5] D. G. Avraam and A. C. Payatakes, Flow regimes and relative permeabilities during steady-state 2-phase flow in porous media, J. Fluid Mech. 293, 207 (1995).

[6] W. L. Olbricht, Pore-scale prototypes of multiphase flow in porous media, Annu. Rev. Fluid Mech. 28, 187 (1996).

[7] S. van der Graaf, T. Nisisako, C. G. P. H. Schroën, R. G. M. van der Sman, and R. M. Boom, Lattice Boltzmann simulations of droplet formation in a T-shaped microchannel, Langmuir 22, 4144 (2006).

[8] G. F. Christopher and S. L. Anna, Microfluidic methods for generating continuous droplet streams, J. Phys. D 40, R319 (2007).

[9] R. Lenormand, C. Zarcone, and A. Sarr, Mechanisms of the displacement of one fluid by another in a network of capillary ducts, J. Fluid Mech. 135, 337 (1983).

[10] A. G. Yiotis, L. Talon, and D. Salin, Blob population dynamics during immiscible two-phase flows in reconstructed porous media, Phys. Rev. E 87, 033001 (2013).

[11] K. T. Tallakstad, H. A. Knudsen, T. Ramstad, G. Løvoll, K. J. Måløy, R. Toussaint, and E. G. Flekkøy, Steady-State Two-Phase Flow in Porous Media: Statistics and Transport Properties, Phys. Rev. Lett. 102, 074502 (2009).

[12] I. Chatzis and F. A. L. Dullien, Dynamic immiscible displacement mechanisms in pore doublets: Theory versus experiment, J. Colloid Interface Sci. 91, 199 (1983).

[13] O. Vizika, D. G. Avraam, and A. C. Payatakes, On the role of viscosity ration during low-capillary number forced imbibition in porous media, J. Colloid Interface Sci. 165, 386 (1994).

[14] T. Chevalier, D. Salin, L. Talon, and A. G. Yiotis, History effects on nonwetting fluid residuals during desaturation flow through disordered porous media, Phys. Rev. E 91, 043015 (2015).

[15] K. T. Tallakstad, G. Løvoll, H. A. Knudsen, T. Ramstad, E. G. Flekkøy, and K. J. Måløy, Steady-state, simultaneous two-phase flow in porous media: An experimental study, Phys. Rev. E 80, 036308 (2009).

[16] S. S. Datta, J.-B. Dupin, and D. A. Weitz, Fluid breakup during simultaneous two-phase flow through a three-dimensional porous medium, Phys. Fluids 26, 062004 (2014).

[17] S. E. Powers, L. M. Abriola, and W. J. Weber, An experimental investigation of nonaqueous phase liquid dissolution in saturated subsurface systems: Steady state mass transfer rates, Water Resour. Res. 28, 2691 (1992).

[18] S. E. Powers, L. M. Abriola, and W. J. Weber, An experimental investigation of nonaqueous phase liquid dissolution in saturated subsurface systems: Transient mass transfer rates, Water Resour. Res. 30, 321 (1994). 
[19] R. I. Al-Raoush and C. S. Willson, A pore-scale investigation of a multiphase porous media system, J. Contam. Hydrol. 77, 67 (2005).

[20] S. S. Datta, T. S. Ramakrishnan, and D. A. Weitz, Mobilization of a trapped non-wetting fluid from a three-dimensional porous medium, Phys. Fluids 26, 022002 (2014).

[21] A. Georgiadis, S. Berg, A. Makurat, G. Maitland, and H. Ott, Pore-scale micro-computed-tomography imaging: Nonwetting-phase cluster-size distribution during drainage and imbibition, Phys. Rev. E 88, 033002 (2013).

[22] R. T. Armstrong, J. E. McClure, M. A. Berrill, M. Rücker, S. Schlüter, and S. Berg, Beyond Darcy's law: The role of phase topology and ganglion dynamics for two-fluid flow, Phys. Rev. E 94, 043113 (2016).

[23] S. Iglauer, S. Favretto, G. Spinelli, G. Schena, and M. J. Blunt, X-ray tomography measurements of power-law cluster size distributions for the nonwetting phase in sandstones, Phys. Rev. E 82, 056315 (2010).

[24] S. Iglauer, M. A. Fernø, P. Shearing, and M. J. Blunt, Comparison of residual oil cluster size distribution, morphology and saturation in oil-wet and water-wet sandstone, J. Colloid Interface Sci. 375, 187 (2012).

[25] R. Oughanem, S. Youssef, D. Bauer, Y. Peysson, E. Maire, and O. Vizika, A multi-scale investigation of pore structure impact on the mobilization of trapped oil by surfactant injection, Transp. Porous Media 109, 673 (2015).

[26] K. Singh, H. Menke, M. Andrew, Q. Lin, C. Rau, M. J. Blunt, and B. Bijeljic, Dynamics of snap-off and pore-filling events during two-phase fluid flow in permeable media, Sci. Rep. 7, 5192 (2017).

[27] S. Sinha and A. Hansen, Effective rheology of immiscible two-phase flow in porous media, Europhys. Lett. 99, 44004 (2012).

[28] A. C. Payatakes, K. M. Ng, and R. W. Flumerfelt, Oil ganglion dynamics during immiscible displacement: Model formulation, AIChE J. 26, 430 (1980).

[29] G. N. Constantinides and A. C. Payatakes, Network simulation of steady-state two-phase flow in consolidated porous media, AIChE J. 42, 369 (1996).

[30] H. A. Knudsen, E. Aker, and A. Hansen, Bulk flow regimes and fractional flow in 2D porous media by numerical simulations, Transp. Porous Media 47, 99 (2002).

[31] H. A. Knudsen and A. Hansen, Two-phase flow in porous media: Dynamical phase transition, Eur. Phys. J. B 49, 109 (2006).

[32] K. M. Ng and A. C. Payatakes, Stochastic simulation of the motion, breakup and stranding of oil ganglia in water-wet granular porous media during immiscible displacement, AIChE J. 26, 419 (1980).

[33] P. Amili and Y. C. Yortsos, Darcian dynamics: A new approach to the mobilization of a trapped phase in porous media, Transp. Porous Media 64, 25 (2006).

[34] M. S. Valavanides and A. C. Payatakes, True-to-mechanism model of steady-state two-phase flow in porous media, using decomposition into prototype flows, Adv. Water. Resour. 24, 385 (2001).

[35] M. S. Valavanides, Review of steady-state two-phase flow in porous media: Independent variables, universal energy efficiency map, critical flow conditions, effective characterization of flow and pore network, Transp. Porous Media 123, 45 (2018).

[36] M. Tyagi and P. Jenny, Probability density function approach for modeling multi-phase flow with ganglia in porous media, J. Fluid Mech. 688, 219 (2011).

[37] L. Talon, N. Goyal, and E. Meiburg, Variable density and viscosity, miscible displacements in horizontal hele-shaw cells. Part 1. Linear stability analysis, J. Fluid Mech. 721, 268 (2013).

[38] T. Chevalier and L. Talon, Generalization of Darcy's law for Bingham fluids in porous media: From flow-field statistics to the flow-rate regimes, Phys. Rev. E 91, 023011 (2015).

[39] M. J. Oak, L. E. Baker, and D. C. Thomas, Three-phase relative permeability of Berea sandstone, J. Pet. Technol. 42, 1054 (1990).

[40] X. He, S. Chen, and R. Zhang, A lattice Boltzmann scheme for incompressible multiphase flow and its application in simulation of Rayleigh-Taylor instability, J. Comput. Phys. 152, 642 (1999).

[41] E. S. Kikkinides, A. G. Yiotis, M. E. Kainourgiakis, and A. K. Stubos, Thermodynamic consistency of liquid-gas lattice Boltzmann methods: Interfacial property issues, Phys. Rev. E 78, 036702 (2008).

[42] T. Lee and C. L. Lin, A stable discretization of the lattice Boltzmann equation for simulation of incompressible two-phase flows at high density ratio, J. Comput. Phys. 206, 16 (2005). 
[43] T. Lee and P. F. Fischer, Eliminating parasitic currents in the lattice Boltzmann equation method for nonideal gases, Phys. Rev. E 74, 046709 (2006).

[44] E. Olsson and G. Kreiss, A conservative level set method for two phase flow, J. Comput. Phys. 210, 225 (2005).

[45] H. Liu, A. J. Valocchi, C. Werth, Q. Kang, and M. Oostrom, Pore-scale simulation of liquid $\mathrm{CO}_{2}$ displacement of water using a two-phase lattice Boltzmann model, Adv. Water Resour. 73, 144 (2014).

[46] A. Fakhari, Y. Li, D. Bolster, and K. T. Christensen, A phase-field lattice Boltzmann model for simulating multiphase flows in porous media: Application and comparison to experiments of $\mathrm{CO}_{2}$ sequestration at pore scale, Adv. Water Resour. 114, 119 (2018).

[47] P. M. Adler, C. G. Jacquin, and J. A. Quiblier, Flow in simulated porous media, Int. J. Multiphase Flow 16, 691 (1990).

[48] M. E. Kainourgiakis, Th. A. Steriotis, E. S. Kikkinides, G. Ch. Charalambopoulou, J. D. F. Ramsay, and A. K. Stubos, Combination of small angle neutron scattering data and mesoscopic simulation techniques as a tool for the structural characterization and prediction of properties of bi-phasic media, Chem. Phys. 317, 298 (2005).

[49] See Supplemental Material at http://link.aps.org/supplemental/10.1103/PhysRevFluids.4.114302 for the complete video animations of the simulated phase distribution dynamics for the Bo values reported in Fig. 3.

[50] J. Hoshen and R. Kopelman, Percolation and cluster distribution cluster multiple labeling technique and critical concentration algorithm, Phys. Rev. B 14, 3438 (1976).

[51] S. S. Datta, H. Chiang, T. S. Ramakrishnan, and D. A. Weitz, Spatial Fluctuations of Fluid Velocities in Flow Through a Three-Dimensional Porous Medium, Phys. Rev. Lett. 111, 064501 (2013).

[52] L. Talon and D. Bauer, On the determination of a generalized Darcy equation for yield-stress fluid in porous media using a Lattice-Boltzmann TRT scheme, Eur. Phys. J. E 36, 139 (2013). 